\title{
Case Presentation: The Effect of Volumetric Image Guidance \& Adaptive Radiotherapy on Cardiac Dose in A Patient with Esophageal Cancer
}

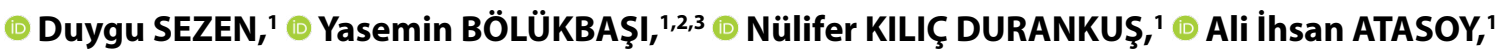 \\ (1) Yücel SAĞLAM, ${ }^{2} \odot$ Vildan ALPAN, ${ }^{2} \odot$ Uğur SELEK ${ }^{1,2,3}$
}

'Department of Radiation Oncology, Koç University, School of Medicine, Istanbul-Turkey

${ }^{2}$ Department of Radiation Oncology, American Hospital, UT MD Anderson Radiation Treatment Center, Istanbul-Turkey

${ }^{3}$ Department of Radiation Oncology, HospitalUniversity of Texas M.D. Anderson Cancer Center, Houston,TX-ABD

\section{Introduction}

Neoadjuvant or definitive chemoradiotherapy constitutes the main approach in the treatment of locally advanced esophageal cancer.[1-3] We present a patient with esophageal cancer whose tumor size regression prompted re-planning to decrease the cardiac dose.

\begin{abstract}
SUMMARY
We present the case of a patient with esophageal cancer whose tumor size regression prompted re-planning to decrease the cardiac dose. A 68-year-old male presented at our outpatient clinic with dysphagia. He was diagnosed with clinical T3N1M0 adenocarcinoma located at the distal esophagus-esophagogastric junction. He was decided to have surgery after receiving neoadjuvant chemoradiotherapy. Following 4-D CT simulation, IG-IMRT with SIB technique was planned as $50 \mathrm{~Gy}$ in 25 fractions to iGTV and as $45 \mathrm{~Gy}$ to the area identified as the CTV. Daily $\mathrm{kV}$ and weekly CBCT were planned at the beginning of the treatment. Concurrent CT with weekly paclitaxel-carboplatin was administered. At the simulation and start of the treatment, the heart was pushed anteriorly due to the mass effect and dilatation in the mid-lower esophagus. The mass and dilatation regressed at the weekly CBCT of the patient. The thirdweek СBCT evaluation revealed the movement of the heart posteriorly into the PTV. Re-simulation was performed to continue with the adaptive planning for the last 10 treatment fractions. The cumulative dose received by the heart was reduced from $96 \%$ to $93 \%$ for V5Gy, from $79 \%$ to $60.8 \%$ for V10Gy, from $60 \%$ to $43.2 \%$ for V15Gy, from $35 \%$ to $21 \%$ for V20Gy, and from 29.6 to $28 \mathrm{~Gy}$ for the mean cardiac dose with the volumetric image-guided adaptive planning. If tumor regression is predicted during radiotherapy to possibly change doses of organs at risk, volumetric image guidance should be encouraged once per week, at least, to consider adaptive treatment when required to ensure the critical organ doses within safe limits.

Keywords: Adaptive radiotherapy; esophageal cancer; volumetric image guidance.

Copyright $\odot$ 2018, Turkish Society for Radiation Oncology
\end{abstract}

\section{Case Report}

A 68-year-old male with no significant past medical history presented at our outpatient clinic with gradually aggravated dysphagia mainly to solids and loss of appetite. He had a history of social alcohol consumption for nearly 40 years but no history of cigarette smoking. 


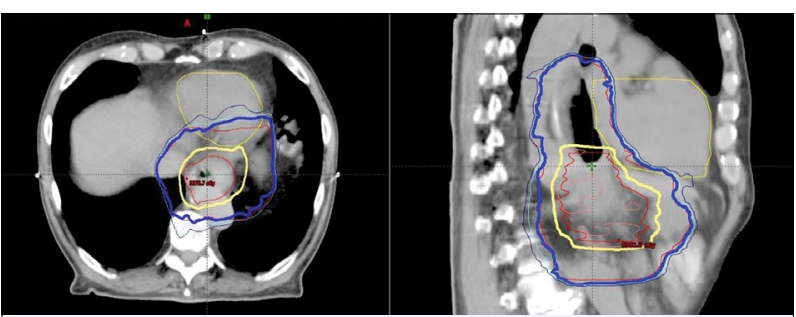

Fig. 1. Initial treatment plan.

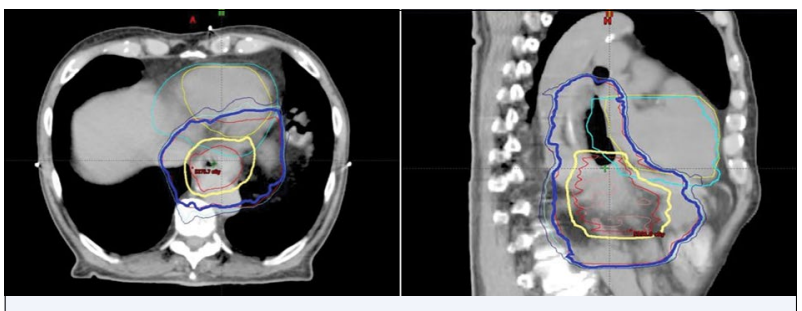

Fig. 2. Initial treatment plan. Pretreatment position of heart in the third week of the treatment (blue contur).

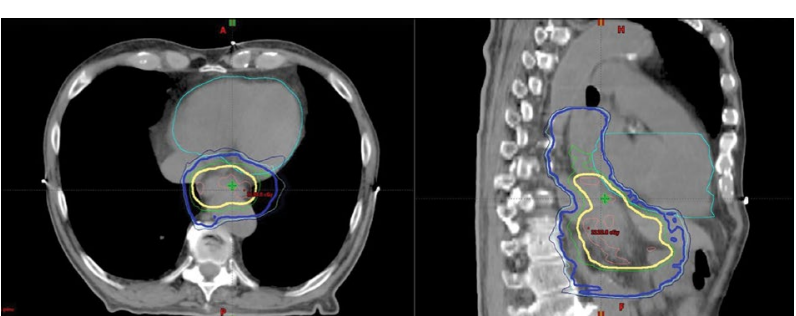

Fig. 3. Adaptive plan on computed tomography re-simulation.

He had a good physical status, with a body mass index of $22 \mathrm{~kg} / \mathrm{m}^{2}$, but he also complained of weight loss of approximately $20 \mathrm{~kg}$ over the last 6 months. He denied any history of nausea, vomiting, abdominal pain, hematemesis, or melena.

His physical examination was unremarkable. There were no lesions in the gingiva, buccal mucosa, floor of the mouth, oral tongue, base of the tongue, hard palate, soft palate, tonsillar fossa, or posterior oropharyngeal soft palate, tonsillar fossa, or posterior oropharyngeal
wall by visualization. On routine investigation, the hewall by visualization. On routine investigation, the he-
moglobin level was $11.9 \mathrm{~g} / \mathrm{dL}$, white blood cell count moglobin level was $11.9 \mathrm{~g} / \mathrm{dL}$, white blood cell count
was $4.7 \mathrm{~K} / \mathrm{uL}$, platelet count was $219 \mathrm{~K} / \mathrm{uL}$, serum creatinine level was $0.92 \mathrm{mg} / \mathrm{dL}$, alanine transaminase level was $56 \mathrm{U} / \mathrm{L}$, and aspartate transaminase level was $13 \mathrm{U} / \mathrm{L}$.

A contrast-enhanced computed tomography (CT) of the thorax and upper abdomen revealed circumferential wall thickening in the lower one-third of the esophagus, causing near-complete lumen occlusion with proximal dilatation as well as enlarged perigastric lymph nodes.

Upper gastrointestinal tract endoscopy revealed an ulcerovegetative tumor of $36 \mathrm{~cm}$ starting from the incisors and extending distally to the inside of the stomach while surrounding the cardia. The upper and middle esophagi were reported to be dilated secondary to the mass. A biopsy of the lesion confirmed moderately differentiated adenocarcinoma.

Staging positron emission tomography with CT defined avid uptake in delayed images extending between the distal part of the esophagus and lesser curvature with a maximum standard uptake value (SUVmax: 26.75). Multiple hypermetabolic lymph nodes were observed adjacent to the cardia and lesser curvature side of the junction, the greatest of which measured $10 \times 15$ fiction, the greatest of which measured $10 \times 15$ $\mathrm{mm}$, whe

Taking all these findings into consideration, our patient was diagnosed with clinical T3N1M0 adenocarcinoma located at the distal esophagus-esophagogastric junction. His case was discussed in a meetin of the Multidisciplinary Oncology Board, and it was decided that he would undergo surgery after receivin neoadjuvant concomitant chemoradiotherapy. He was also referred to a dietetic team because of his weigh loss and was advised to modify his diet. Following 4-dimensional CT simulation, image-guided intensitymodulated radiotherapy (IG-IMRT) with simultaneous integrated boost technique was planned as $50 \mathrm{~Gy}$ in 25 fractions ( $2 \mathrm{~Gy} /$ day) to the primary disease, defined as the internal gross tumor volume, and as $45 \mathrm{G}$ $(1.8 \mathrm{~Gy} /$ day) to the area identified as the clinical tumo volume. The plan was generated with $0^{\circ}, 40^{\circ}, 80^{\circ}, 120^{\circ}$ $160^{\circ}$. The plan was generated with $0^{\circ}, 40^{\circ}, 80^{\circ}, 120^{\circ}$ arrangements. The simulatio 列 performed after 3 hours of fasting in order to provide simila gastic dimensions. Daily $k V$ and weekly cone beam $\mathrm{CT}$ (CBCI) was planned at the beginning of the treatment. Concurrent chemotherapy with weekl paclitaxel $60 \mathrm{mg} / \mathrm{m}^{2}$-carboplatin AUC 2 was administered by the medical oncology department

At the simulation and start of the treatment, the heart was pushed anteriorly due to the mass effect an dilatation in the mid-lower esophagus. The mass and dilatation regressed at the weekly volumetric imageguided evaluation $(\mathrm{CBCT})$ of the patient during the treatment process. The third-week CBCT evaluation revealed the movement of the heart posteriorly into the planning treatment volume (PTV). The magnitude of the regression was $<0.2 \mathrm{~cm}$ in the first week but the largest regression $0 \mathrm{cur} 2 \mathrm{~cm}$ in the fisterior-poster dimento from the second to the third week as 1.4 $\mathrm{cm}$ in total due to the regression of the esophagus mass and dilatation.

In light of this major finding, re-simulation and replanning were performed to continue with the adaptive planning for the last 10 treatment fractions without any treatment break. Compared with the previously planned values, the dose received by the heart for the last 10 fractions was decreased from $96 \%$ to $90 \%$ for V5Gy, from $60 \%$ to $47 \%$ for V10Gy, from $30 \%$ to $18 \%$ for V15Gy, from $8 \%$ to $0 \%$ for V20Gy, and from 11.82 to $10.38 \mathrm{~Gy}$ for the mean cardiac dose. The heart V5Gy value was $100 \%$, V10Gy value was $70 \%, \mathrm{~V} 15 \mathrm{~Gy}$ value was $60 \%$, V20Gy value was $35 \%$, and the mean cardiac dose was $17.72 \mathrm{~Gy}$ for the initial 15 fractions. Within this framework, the cumulative dose received by the heart calculated in the comperte plan, was reduced heart, calcula from $96 \%$ to $93 \%$ in V10Gy, fon $60 \%$ to $43.2 \%$ for V15Gy, from $35 \%$ to $21 \%$ for $\mathrm{V} 20 \mathrm{~Gy}$, and from 29.6 to $28 \mathrm{~Gy}$ for the mean cardiac dose with the volumetric image-guided adaptive planning (Figs.)

At the end of the radiotherapy, his dysphagia was partially relieved. The treatment was completed without any unexpected complications or acute side effects.

Discussion

Radiotherapy has an important role in both operable and inoperable esophageal cancer. However, radiotherapy planning for esophageal cancer has difficulties due to the central location of the esophagus; large treatment volumes concerning the risk of transmural and lymphatic spread; and the proximity of critical organs, such as the heart, lungs, and spinal cord.

Advances in radiation techniques have resulted in higher treatment response rates, more normal tissue sparing, and less treatment time. Recently, IG-IMRT has replaced 3-dimensional conformal radiotherapy for esophageal cancer owing to relatively preferable for esophageal cancer owing to relatively preferable
dose distributions as well as significant dose reductions dose distributions as well as significant dose reductions in critical organs. The current literature rationalizes the increasing use of IMRT.[4-6] In contrast, IMRT requires more precise mechanisms in the planning and treatment process with regard to smaller safety margins. Thus, 4-dimensional CT is useful to consider movements secondary to respiration, circulation, or peristalsis.[7-9] Volumetric-modulated arc treatment is also advantageous with shorter treatment times that may decrease the risk of intrafractional positional shifts. [10]

In addition to intrafractional chances, interfracInal differences may occur due to tumor regression, progression, or displacement. In this context, СВСТ is an inevitable component of modern radiotherapy procedures. Planar $\mathrm{kV}$ imaging remains incapable in organs without bony structures, such as the esophagus. Martins et al. compared planar kV imaging versu CBCT in the evaluation of setup errors in esophagu carcinoma radiotherapy [11]; sixteen patients, $212 \mathrm{kV}$ images, and 116 CBCT images were reviewed in that study, revealing superiority of CBCT over planar $\mathrm{kV}$ imaging due to the soft tissue structure of the esophagus and a decrease in the number of possible setup errors with $\mathrm{CBCT}$. There was no additional significant relationship between setup errors and immobilization system or tumor location. Although a small sample size was a limitation of that study, routine-use $\mathrm{CBCT}$ was encouraged by the authors [11]

To date, there have been limited studies addressing esophageal displacement as a numerical value during radiotherapy. Yamashita et al. analyzed the shift of the esophagus in 20 patients treated with radiotherapy for esophageal cancer.[12] CBCT was performed for each patient twice a week. According to the results, the authors suggested the use of target margins of $9 \mathrm{~mm}$ for day-to-day esophageal motion and $8 \mathrm{~mm}$ for patien setup in all directions, respectively [12]; if $\mathrm{CBCT}$ is a standard procedure for daily imaging, setup errors may be neglected and only day-to-day esophageal motion of $9 \mathrm{~mm}$ may be taken into account. Although the results of the present study are consistent with those of previous studies [13], mentioned margins raise the question of whether we need to use $\mathrm{CBCT}$ more frequently than twice a week

Online volumetric images of the patient in the treatment position provide further information about current status, and regular volumetric imaging during the treatment process enables the evaluation of the necessity of adptive planning. Hawkins et al. evaluethe organs at risk for 14 cases by creating a patientThe

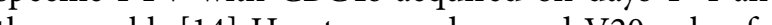
then were signifenty the decreased 政 the first week of treatment using CBCT. In a similar trial, adaptive plans were created based on CBCTs that were acquired daily for the first week and then weekly. [15] Adaptive plans revealed significantly reduced V10Gy, V20Gy, and mean lung dose values as well as smaller D35\% and mean heart doses compared with the initial plans.[15] In another study from Denmark, 29 patients with esophageal cancer were evaluated with daily $\mathrm{CBCT}$ and an additional $\mathrm{CT}$ at medial fraction 10 (range, 9-14). [16] After the contouring and re-planning processes, two CTs were compared whether there 
was a decrease $<1 \%$ in CTV or $<3 \%$ in PTV coverage that was accepted. In case of a larger decrease, previous CBCTs were reviewed in terms of adaptive planning requirements. Nine of the 29 patients underwent adaptive radiotherapy, and an increased V30Gy dose to the heart of $>2 \%$ was observed in nine (31\%) patients (maximum 5\% increase). Although this result is consistent with that of the presented case, there also exists conflicting data regarding the heart dose.[17]

As adaptive planning has become widespread, questions have been raised regarding the incidence of local recurrence of the adaptive treatment and particulacly recurence of the in the are excluded wish the risk for fallure in the area excluce with subsequent planning. The linited number of publications has thus far investigated the effect of local failure patterns concerning thoracic tumors, and the greater part of them is related to lung cancer. Ramella et al. prospectively analyzed 50 patients with locally advanced non-smallcell lung cancer treated with concomitant chemoradiotherapy.[18] The patients underwent weekly CT simulation during treatment. Adaptive planning was outlined in cases of tumor regression. Patterns of failure were classified as in-field (progression within the re-planning PTV), marginal (recurrence in the initial PTV excluded from the re-planning PTV), and out-of feld (recurrece outside of the initial PTV). Mart field (recurrence our relapse was recorded in $6 \%$ of the patients, whereas $20 \%$ and $4 \%$ of the patients showed in-field and out-offield local failure, respectively. Ramella et al. deserves appreciation for drawing attention to the failure patterns after adaptive radiotherapy.

\section{Conclusion}

In the evolution of radiation oncology, there is an ongoing effort to increase the treatment efficiency without any major acute and long-term toxicity. The current literature supports the use of IMRT, including 4-D imaging, for esophageal cancer. Nevertheless, target and organs at risk motion might be a major problem in esophageal radiotherapy, and the importance of volumetric image guidance and adaptive planning if necessary, sound to becritical Overall, if tumor regression

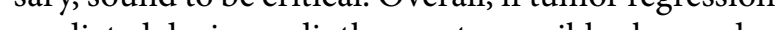
predicted during radiotherapy to possibly change doses

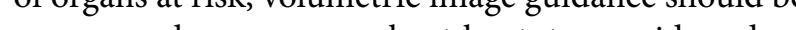
encouraged once per week, at least, to consider adaptive treatment when required to ensure critical organ doses within safe limits.

Peer-review: Externally peer-reviewed.

Conflict of Interest: The authors declare that there is no conflict of interest.
Authorship contributions: Concept - D.S.; Design A.I.A.; Supervision - V.A.; Data collection \&/or processing - N.K.D.; Analysis and/or interpretation - Y.B.;

\section{References}

1. van Hagen P, Hulshof MC, van Lanschot JJ, Steyerberg EW, van Berge Henegouwen MI, Wijnhoven BP et al; CROSS Group. Preoperative chemoradiotherapy for esophageal or junctional cancer. N Engl J Med 2012;366(22):2074-84

2. Cooper JS, Guo MD, Herskovic A, Macdonald JS, Martenson JA Jr, Al-Sarraf M, et al. Chemoradiotherapy of locally advanced esophageal cancer: long-term follow-up of a prospective randomized trial (RTOG 85-01). Radiation Therapy Oncology Group. JAMA 1999;281(17):1623-7.

3. Tepper J, Krasna MJ, Niedzwiecki D, Hollis D, Reed $\mathrm{CE}$, Goldberg R, et al. Phase III trial of trimodality therapy with cisplatin fluorouracil, radiotherapy and surgery compared with suroucry a lone for erapy, an cancer 92.

4. Kole TP, Aghayere O, Kwah J, Yorke ED, Goodman KA. Comparison of heart and coronary artery dose associated with intensity-modulated radiotherapy versus three-dimensional conformal radiotherapy for distal esophageal cancer. Int J Radiat Oncol Biol Phys 2012;83(5):1580-6

5. Münch S, Aichmeier S, Hapfelmeier A, Duma MN, Oechsner M, Feith M, et al. Comparison of dosimetric parameters and toxicity in esophageal cancer patient undergoing 3D conformal radiotherapy or VMAT. Strahlenther Onkol 2016;192(10):722-9.

6. Xu C, Xi M, Komaki R, Balter PA, Huang M, Hobbs $\mathrm{BP}$, et al. Dosimetric and clinical outcomes after volumetric modulated arc therapy for carcinoma of the thoracic esophagus. Adv Radiat Oncol 2017;2(3):325-

7. Hu CY, Li JB, Wang JZ, Wang W, Li FX, Guo YL. Comparison of gross tumor volume of primary oesophageal cancer based on contrast-enhanced three-dimensional, four-dimensional, and cone beam computed tomography. Oncotarget 2017;8(56):95577-85.

8. Dieleman EM, Senan S, Vincent A, Lagerwaard F Slotman BJ, van Sörnsen de Koste JR. Four-dimensional computed tomographic analysis of esophagea mobility during normal respiration. Int J Radiat Onco Biol Phys 2007;67(3):775-80.

9. Patel AA, Wolfgang JA, Niemierko A, Hong TS, Yock $\mathrm{T}$, Choi NC. Implications of respiratory motion as measured by four-dimensional computed tomography for radiation treatment planning of esophageal cancer. Int I Radiat Oncol Biol Phys 2009;74(1):290-6.

10. Vivekanandan N, Sriram P, Kumar SA, Bhuvaneswari N, Saranya K. Volumetric modulated arc radiotherapy for esophageal cancer. Med Dosim 2012;37(1):108-13

11. Martins L, Couto JG, Barbosa B. Use of planar kV vs. CBCT in evaluation of setup errors in oesophagus carcinoma radiotherapy. Rep Pract Oncol Radiother 2016;21(1):57-62.

12. Yamashita H, Haga A, Hayakawa Y, Okuma K, Yoda $\mathrm{K}$ Okano $\mathrm{Y}$ et al. Patient setup error and day-to-day esopheal motion error analyzed by cone-beam compuled puted tomography in radiation therapy. Acta Oncol
2010;4(4):485-90.

13. Cohen RJ, Paskalev K, Litwin S, Price RA Jr, Feigenberg SJ, Konski AA. Esophageal motion during radiotherapy: quantification and margin implications. Dis Esophagus 2010;23(6):473-9.

14. Hawkins MA, Brooks C, Hansen VN, Aitken A, Tait DM. Cone beam computed tomography-derived adaptive radiotherapy for radical treatment of esophageal cancer. Int J Radiat Oncol Biol Phys 2010;77(2):378-

15. Sriram P, Syamkumar SA, Kumar JS, Prabakar S, Dhanabalan R, Vivekanandan N. Adaptive volumetric modulated arc treatment planning for esophageal cancers using cone beam computed tomography. Phys Med 2012;28(4):327-32.

16. Nyeng TB, Nordsmark M, Hoffmann L. Dosimetric evaluation of anatomical changes during treatment to identify criteria for adaptive radiotherapy in oesophageal cancer patients. Acta Oncol 2015;54(9):1467-73.

17. Wang J, Lin SH, Dong L, Balter P, Mohan R, Komaki R, et al. Quantifying the interfractional displacement of the gastroesophageal junction during radiation therapy for esophageal cancer. Int $J$ Radiat Oncol Biol Phys 2012;83(2):e273-80.

18. Ramella S, Fiore M, Silipigni S, Zappa MC, Jaus M, Alberti AM, et al. Local Control and Toxicity of Adaptive Radiotherapy Using Weekly CT Imaging: Results from the LARTIA Trial in Stage III NSCLC. J Thorac Oncol 2017;12(7):1122-30. 